\title{
Uncultured microorganisms as a source of secondary metabolites
}

\author{
Kim Lewis ${ }^{1}$, Slava Epstein ${ }^{2}$, Anthony D'Onofrio ${ }^{1}$ and Losee L Ling ${ }^{3}$ \\ The vast majority of microbial species are 'uncultured' and do not grow under laboratory conditions. This has led to the \\ development of a number of methods to culture these organisms in a simulated natural environment. Approaches include \\ placing cells in chambers that allow diffusion of compounds from the natural environment, traps enclosed with porous \\ membranes that specifically capture organisms forming hyphae-actinobacteria and microfungi, and growth in the presence of \\ cultivable helper species. Repeated cultivation in situ produces domesticated variants that can grow on regular media in vitro, \\ and can be scaled up for secondary metabolite production. The co-culture approach has led to the identification of the first class \\ of growth factors for uncultured bacteria, iron-chelating siderophores. It appears that many uncultured organisms from diverse \\ taxonomical groups have lost the ability to produce siderophores, and depend on neighboring species for growth. The new \\ cultivation approaches allow for the exploitation of the secondary metabolite potential of the previously inaccessible \\ microorganisms.
}

The Journal of Antibiotics (2010) 63, 468-476; doi:10.1038/ja.2010.87; published online 21 July 2010

Keywords: antibiotic; biofilm; cultivation; siderophore; uncultivable; unculturable; uncultured

\section{INTRODUCTION}

Celebrating its 112th anniversary, the Great Plate Count Anomaly is arguably the oldest unresolved microbiological phenomenon. An Austrian microbiologist Heinrich Winterberg made a curious observation in 1898: the number of microbial cells in his samples did not match the number of colonies formed on nutrient media. ${ }^{1}$ J Amann quantified this mismatch a decade later, and showed nongrowing cells outnumbered the cultivable ones almost 150 times. $^{2}$ This phenomenon first revealed by these early studies is today known as the Great Plate Count Anomaly. ${ }^{3}$ In the years to follow, the phenomenon was repeatedly confirmed by all microbiologists who cared to compare initial cell counts to colonies formed in Petri dishes. ${ }^{4-6}$ By midcentury, the Great Plate Count Anomaly became a universally recognized phenomenon, acknowledged by several classics of the time. ${ }^{7-9}$

Studies of rRNA detectable in the environment provided a stunning new glimpse of the microbial diversity missed by traditional cultivation methods. ${ }^{10}$ Dozens of novel microbial divisions were discovered. ${ }^{11-19}$ From the molecular surveys of the 1990s a new picture of the environment emerged-one full of uncultured bacterial groups waiting to be isolated. ${ }^{20,21}$ What microbiologists had been able to cultivate and catalogue throughout the entire history of microbiological exploration ${ }^{22}$ appeared to be a small fraction of what awaited cultivation. This gap has proven difficult to close and even today most of the known microbial divisions have no single cultivable representative. $^{23,24}$ This gap seems to be as large now as it was back in
1898. ${ }^{1,5,25}$ The need to close the gap and access novel bacterial species is an urgent practical issue, as biotech and pharmaceutical industries appear to culture and screen microbial taxa that microbiologists seem to be repeatedly reisolating. ${ }^{26}$ Discovering the underlying mechanisms responsible for uncultivability of the microbial majority and developing ways to circumvent limitations of conventional cultivation techniques is recognized as a top research priority for microbial biology. ${ }^{27,28}$ Understanding why uncultivated microorganisms are uncultivated is an important first step to access and use their seemingly infinite diversity.

\section{METHODS FOR GROWING UNCULTURED MICROORGANISMS}

Several terms have been used in the literature, including 'uncultured', 'unculturable' and 'uncultivable' to describe bacteria that are not readily cultured in the lab. Once these uncultured strains are successfully isolated by novel methods, they can be more accurately referred to as 'previously uncultured' bacteria, but this term is often, as is the case in this text, still abbreviated as 'uncultured'. The term uncultured stresses the novel cultivation conditions that were required for initial cultivation.

A recent resurgence in cultivation efforts has been sparked by the vast diversity of uncultured bacterial groups revealed by environmental surveys of 16S rRNA. ${ }^{29-40}$ Although some novel bacterial species were successfully cultured by varying media and growth conditions, ${ }^{41}$ significant departures from conventional techniques were clearly

\footnotetext{
${ }^{1}$ Antimicrobial Discovery Center and Department of Biology, Northeastern University, Boston, MA, USA; ${ }^{2}$ Department of Biology, Northeastern University, Boston, MA, USA and ${ }^{3}$ NovoBiotic Pharmaceuticals, Cambridge, MA, USA

Correspondence: Dr K Lewis, Antimicrobial Discovery Center and Department of Biology, Northeastern University, 134 Mugar Hall, 360 Huntington Avenue, Boston, MA 02115, USA. E-mail: k.lewis@neu.edu 
a

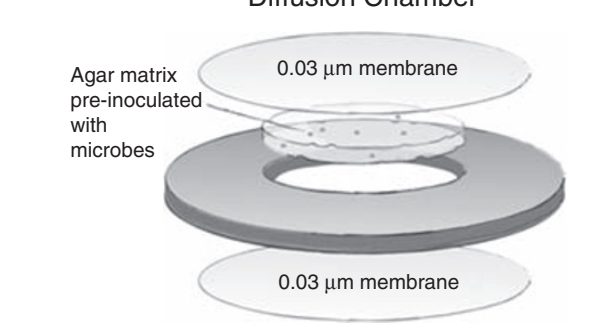

b

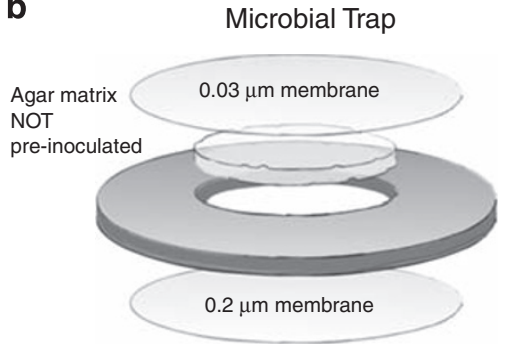

Air

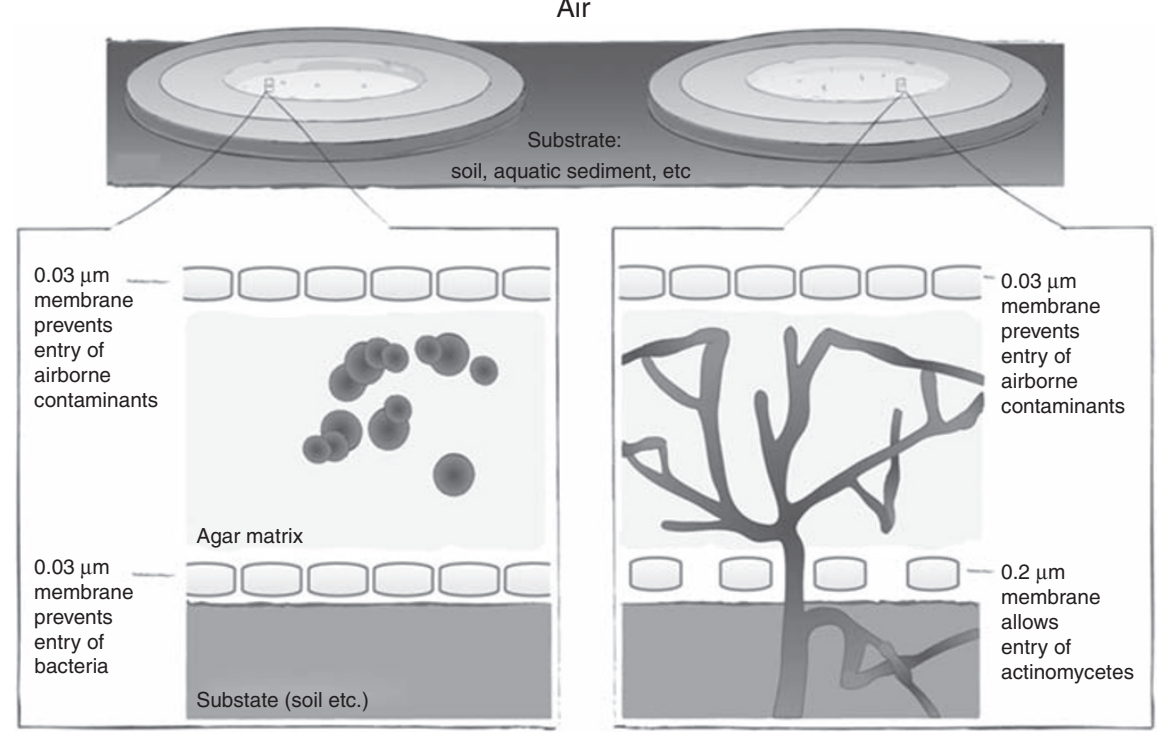

Figure 1 Design and application of the diffusion chamber (a) and the microbial trap (b).

in order, and indeed the new technologies substantially diverged from traditional cultivation methods by adopting single cell and high-throughput strategies, ${ }^{32,37,38,40}$ better mimicking the natural milieu, ${ }^{29,31,34,39}$ increasing the length of incubation and lowering the concentration of nutrients. ${ }^{33}$ High-throughput extinction culturing is based on the dilution of natural communities of bacteria to 1-10 cells per well in low nutrient, filtered marine water. This strategy resulted in the cultivation of the first member of the ubiquitous, previously uncultured clade, SAR11. ${ }^{38}$ Our research group contributed to the effort by developing three cultivation methodologies. ${ }^{35-37}$ All three strategies aim to provide microorganisms with their natural growth conditions by incubating them in simulated natural environments.

The diffusion chamber is designed to essentially 'trick' cells into thinking they are growing in their natural environment by creating an incubation strategy that very closely mimics their natural habitat. ${ }^{35}$ The diffusion chamber consists of a stainless steel washer and $0.03-\mu \mathrm{m}$ pore-size membranes (Figure 1a). After gluing a membrane to one side of the washer, the inoculum (a mix of environmental cells and warm agar) is introduced, and the second membrane seals the chamber. Nutrients from the environment can diffuse into the chamber, therefore it is not necessary to add them to the medium. Once inoculated and assembled, the chamber can be returned to the original location of sampling or in a simulated natural environment such as a block of sediment kept in an aquarium in lab. Microcolonies grow in the chamber during such incubation (Figure 2). We have tested this method with samples from various environments and found it particularly successful in terms of both cell recovery and diversity of cultured isolates.
The first experiments with the diffusion chamber were carried out with microorganisms collected from a sandy tidal flat in Massachusetts Bay and detached from sediment by vortexing. ${ }^{35}$ After $1-3$ weeks of incubation, the chambers were opened and microbial colonies were examined, counted and subcultured by transfer into new chambers (Figure $2 \mathrm{~b}$ ). A recovery rate of $22 \%$ on average was observed in the diffusion chambers, exceeding hundreds fold what could be achieved using standard Petri dishes. In this study and follow-up research ${ }^{30,37}$ we isolated numerous species that did not grow in Petri dishes inoculated with environmental samples, but were successfully grown in the diffusion chambers.

One such isolate, MSC33, became a model object in studying microbial uncultivability. ${ }^{37}$ MSC33 was originally cultured in diffusion chambers and could not be isolated in vitro. We discovered however that it could be co-cultured in standard Petri dishes with another marine isolate, MSC105, which we termed a helper strain. Co-cultured with MSC105, MSC33 eventually formed a domesticated variant MSC33c that could grow in vitro in pure culture. We then discovered that pairing MSC33c with its maternal, and 'uncultivable', strain MSC33 induced the latter to grow. As spent medium conditioned by MSC33c was also shown to induce growth of MSC33, we were able to use bioassay guided fractionation to determine the nature of the growth-inducing factor that proved to be a short peptide. Testing synthetic versions of various lengths of this peptide showed that the 5 amino-acid peptide, LQPEV, had optimal growth induction activity. Maximum activity was observed at $3.5 \mathrm{~nm}$ of the peptide, suggesting its role as a signal as opposed to nutrient.

The diffusion chamber was also used to culture bacteria from sediment from a freshwater pond near Boston. ${ }^{30}$ The chambers were 

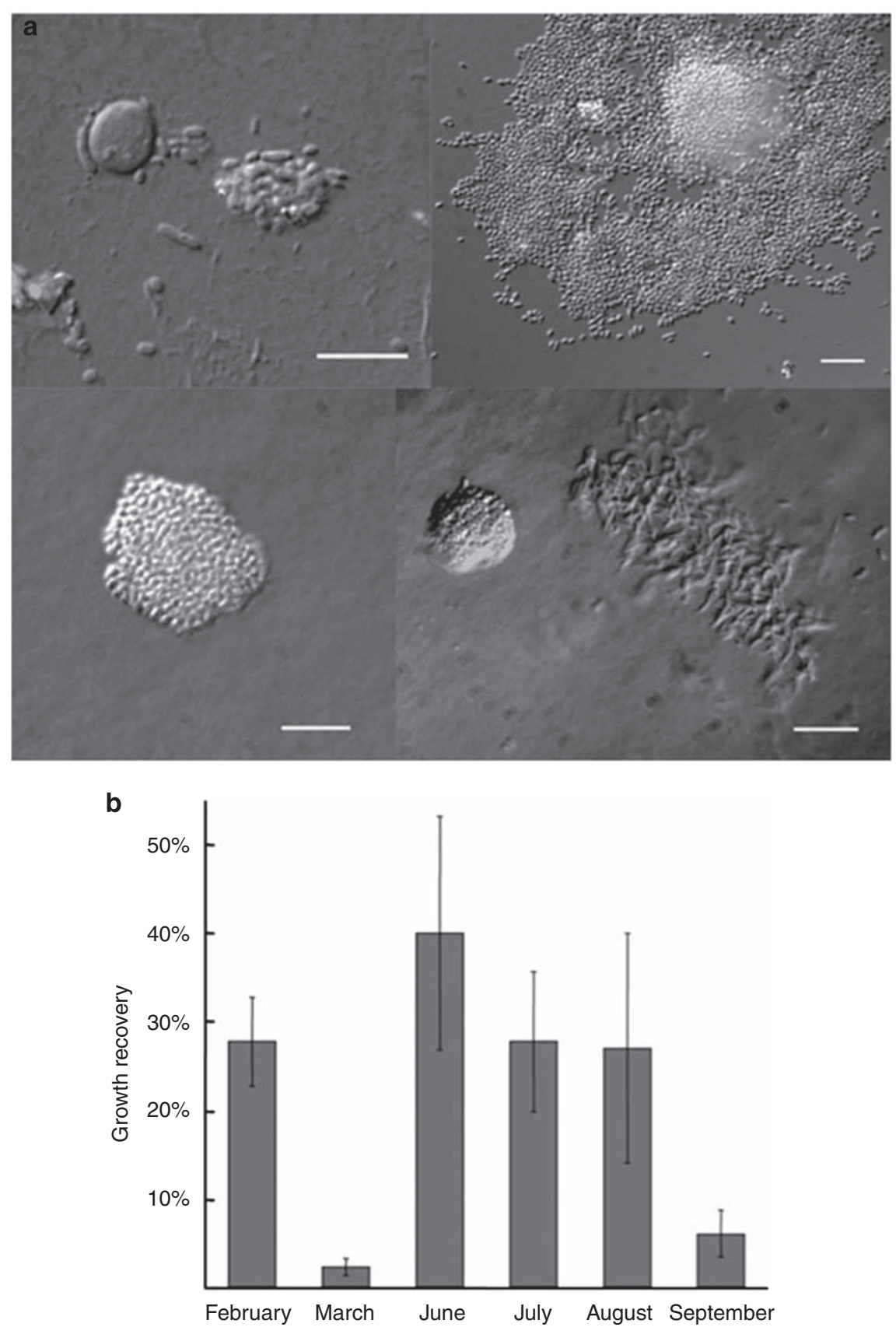

Figure 2 Microcolonies of marine microorganisms grown inside a diffusion chamber incubated in simulated natural environment (a) Bar=5 $\mu m$. Growth recovery of environmental cells in diffusion chambers plotted as percent of inoculated cells forming colonies (b); Kaeberlein et al..$^{35}$

incubated on top of a freshly collected block of sediment kept under a layer of aerated extant water in an aquarium. They were opened after 4 weeks and the material was homogenized with a syringe, diluted with sterile filtered pond water and part of the material was incubated in freshly constructed diffusion chambers. The remaining material was used to prepare standard pour plates supplemented with diluted (10\% of manufacturer recommended) LB broth. Re-incubation in new diffusion chambers was performed once again, to three rounds of in situ cultivation, each time with parallel subculturing in standard Petri dishes. In this way, we could assess if new species would appear by repetitive incubations of the chambers, and what species from thus grown diversity could be grown in vitro. Indeed, $70 \%$ of the 438 strains isolated came from the diffusion-chamber-reared material, and several strains unique to this approach only appeared after two or more rounds of cultivation in situ. Two examples of members of rarely cultivated phyla isolated after repeated cultivation in the diffusion chambers belonged to Verrucomicrobia and Acidobacteria. The overlap with species grown by initially plating on Petri dishes was minimal and only represented $7 \%$ of the species cultured. This confirmed a similar observation made in a different environment ${ }^{37}$ - one or more rounds of growth inside diffusion chambers leads to domestication of novel microbial species that are difficult to grow otherwise.

The diffusion chamber typically produces a mixed culture that requires considerable time to isolate, purify and re-inoculate 
a

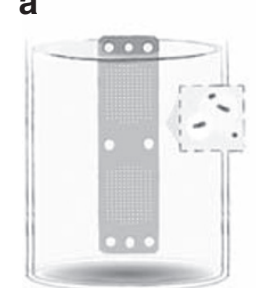

b

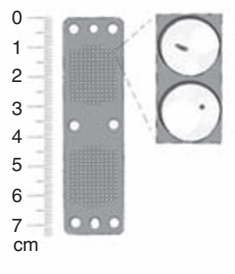

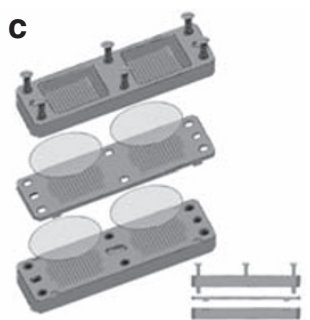

Figure 3 Isolation chip, or ichip, for high-throughput microbial cultivation in situ. Left panel shows dipping a plate with multiple through holes into a suspension of cells such that each hole captures (on average) a single cell (a), as illustrated in the central panel (b). Right panel shows how ichip is assembled: membranes cover arrays of through holes from each side; upper and bottom plates with matching holes press the membranes against the central (loaded) plate. Screws provide sufficient pressure to seal the content of individual through holes, which become miniature diffusion chambers containing single cells (c). (Artwork by Stacie Bumgarner, Whitehead Institute for Biomedical Sciences, Cambridge, MA, USA).

individual colonies. To streamline this process into a high-throughput system, we developed a variant of the diffusion chamber for massively parallel microbial isolation. The Isolation Chip, or ichip for short (Figure 3; Nichols et al. ${ }^{37}$ ), consists of hundreds of miniature diffusion chambers that can be loaded with an average of one cell per chamber. The ichip enables microbial growth and isolation in a single step with hundreds of individual cultures incubating on a single chip.

The ichip consists of a central plate for incubating microbial cells, semipermeable membranes on each side to restrict the motion of cells and side panels that mainly hold the structure together (Figure 3). When the central plate is dipped into a suspension of cells in molten agar (Figure 3a), its through holes capture miniature volumes of this suspension, which solidify in the form of small agar plugs. The membranes, which are fastened by screws, serve to separate each plug from one another as well as the outside environment (Figure 3c). An array of small diffusion chambers is created, each filled with agar and cells. The number of cells in each well can be controlled by adjusting the initial concentration of cells in the molten agar used for inoculation (Figure $3 b$ ). The ichip is then incubated in nature or a simulated version of the original habitat and produces pure cultures in each well that was originally inoculated with a single cell. This design greatly reduces cultivation efforts and allows for high-throughput cultivation of uncultured microorganisms.

In a proof-of-concept study, we used the ichip to grow marine water column and soil microorganisms, recorded the colony count and compared the rRNA gene diversity of ichip-grown microorganisms and their colony count from parallel incubations in standard Petri dishes. ${ }^{42}$ Samples were taken from both seawater and wet soil near the Marine Science Center of Northeastern University, Nahant, MA, USA and disrupted by sonication. Cells were counted with DAPI and then inoculated into ichips after dilution to $10^{3}$ cells per $\mathrm{ml}$ allowing $500 \mu \mathrm{l}$ of cell-agar mix to load $1.25 \mu \mathrm{l}$ into each well with an average of 1.25 cells (Figure 3 ). To approximate the species diversity inoculated into ichips, for conventional cultivation, we established miniature Petri dishes with $500 \mu \mathrm{l}$ of the cell-agar mixes in 24-well culture plates. The ichip chambers were incubated for 2 weeks in their natural environments before observation. Approximately 50 agar plugs from each ichip were removed for detailed microscopic examination and colony counting. The rest was used to isolate DNA for 16S rRNA gene sequence-based identification of grown species. The Petri dish controls were processed in an identical manner.

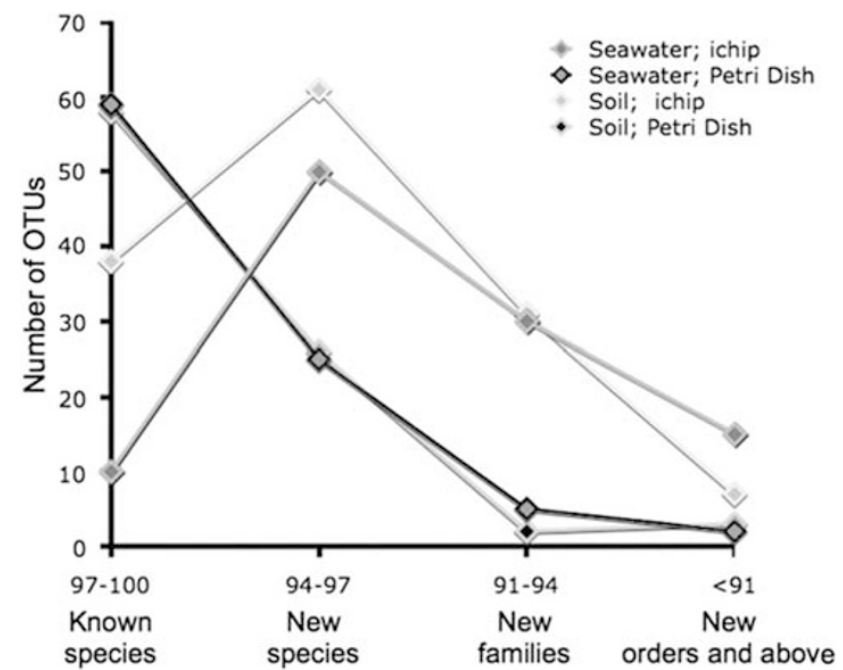

Figure 4 Novelty of seawater and soil microbial strains grown in ichip and Petri dish. Equation of sequence novelty, in percent diversion from the known species, and taxonomic rank of novelty (genus-, family level, etc.) is very approximate.

This study led to three important observations. First, $40-50 \%$ of cells incubated in ichips formed colonies, which exceeded the Petri dish recovery fivefold. Second, the ichip- and Petri dish-grown collections of microorganisms were dramatically different and shared only one species (Vibrio sp.). This lack of overlap between the two species lists was not due to undersampling, because Petri dishes inoculated with seawater and soil samples did share several species, as did ichips inoculated with microorganisms from these two sources. Third, the novelty of isolates grown in ichips significantly exceeded that of Petri dish-reared species (Figure 4). The results indicated that ichip allows the growth of bacterial species from diverse groups, which produce unique collections compared with traditional methods. The method does so in a high-throughput manner that produces relatively large collections with a minimal amount of time and effort.

Microorganisms that are particularly important for drug discovery, microscopic fungi and actinomycetes grow by forming filaments capable of penetrating soft substrates. Because actinomycetes can pass through $0.2 \mu \mathrm{m}$ pores, we reasoned this could be used to design a trap for the specific capture of these organisms (trap method, Figure 1b). ${ }^{36}$ The trap is similar in design to the diffusion chamber, except the membranes have larger pores and the agar inside the trap is initially sterile when placed in the environment (contrast Figures 1a and b). Any growth observed afterwards inside the trap is due to the movement of cells into the trap during incubation.

The trap was tested ${ }^{36}$ by incubating these devices on top of garden soil kept in large Petri dishes, sealed with parafilm to prevent evaporation. The traps were opened after 14-21 days of incubation at room temperature in the dark. The slabs of solid agar or gellan gum were then removed, inverted and placed into a sterile Petri dish. In several experiments, the agar slabs were incubated undisturbed for 5-7 days before colony sampling to allow actinomycetes to form aerial mycelia to facilitate colony picking. Subcultivation was repeated to obtain pure cultures. For comparative purposes, we also prepared parallel conventional Petri dishes by inoculating them with the airdried soil material directly, followed by 2-week-long incubation in the laboratory. Similarly to subculturing trap-derived material, conventional Petri dishes were established with agar or gellan gum, and were supplemented with the same nutrient additives. After incubation and 

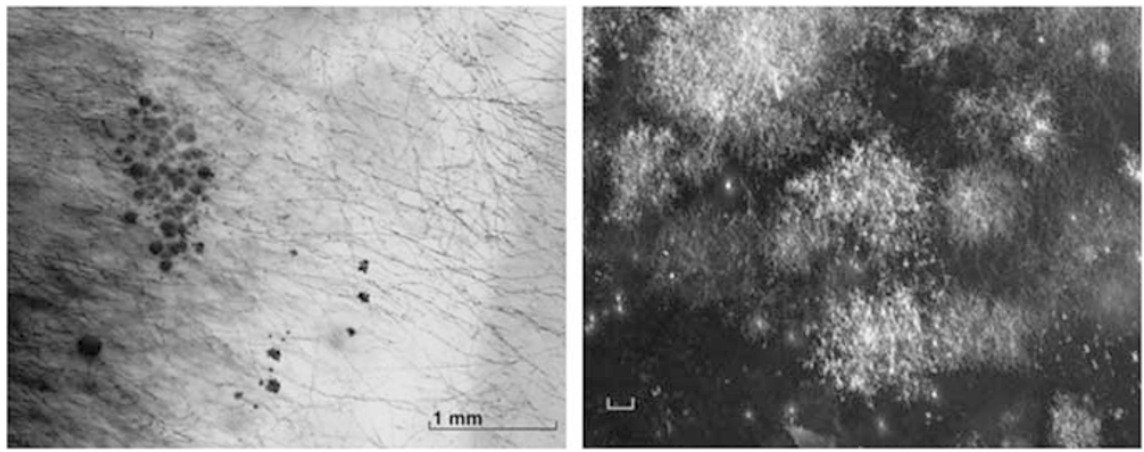

Figure 5 Bacterial colonies and fungal hyphae (left panel) and actinomycetes-like colonies (right panel, scale bar=0.1 mm) grown in a trap after 2 weeks of incubation in garden soil.

purification, 90 colonies from traps and 90 colonies from conventional Petri dishes were identified and compared by sequencing their $16 \mathrm{~S}$ rRNA genes. Visual inspection of traps revealed an abundance of actinobacterial colonies (Figure 5). Traps with 0.4 - to $0.6-\mu \mathrm{m}$ poresized bottom membranes contained a significant number of fungal filaments, but cutting the pore size to $0.2 \mu \mathrm{m}$ effectively excluded fungi. The majority of organisms grown in the traps proved to be actinomycetes, some of which represented rare and unusual species from the genera Dactylosporangium, Catellatospora, Catenulispora, Lentzea and Streptacidiphilus. In general, gellan-gum-based traps appeared to have captured more diverse assemblage of actinomycetes; conventional Petri dishes produced more nonfilamentous actinobacteria. We concluded that the trap method allows for selective capture of filamentous actinomycetes enriched for new and rare species.

\section{CULTIVATION AND DOMESTICATION OF UNCULTURED MICROORGANISMS}

A particularly useful way to gain access to uncultured isolates that initially grow in a diffusion chamber is to select for domesticated variants that become capable of growth on standard media. This domestication process occurs after repeated transfer of colonies grown in a diffusion chamber into new chambers and eventually onto standard media. Domesticated variants of strains grown initially in a diffusion chamber appear after anywhere from one to more than four repeated transfers (Figure 6). ${ }^{37} \mathrm{~A}$ qualitative example is the rich diversity of bacterial species we recovered from the apparently lifeless sands in Dry Valley, Antarctica: once cells from these sands went through a growth cycle in a diffusion chamber incubated within the sand sample. Further experiments more carefully documented the phenomenon of domestication. In a separate study of 'uncultivables' a collection of domesticated strains was isolated by passaging a fresh pond sample through several rounds of diffusion chamber growth. ${ }^{30}$ A substantial difference was noticed when comparing this collection with isolates from samples plated directly on traditional Petri dishes. Isolates unique to the diffusion chamber approach outnumbered the 'traditional' isolates by a factor of 3, with only $7 \%$ of the total 438 isolates shared between the collections. The domesticated pool included representatives of rarely cultivated phyla, such as Deltaproteobacteria, Verrucomicrobia, Spirochaetes and Acidobacteria, whereas the 'traditional' pool did not. Up to three transfers through the diffusion chambers were necessary for some of the more interesting species to form domesticated variants. In a follow-up study of actinobacteria, we again observed a very substantial number of strains appearing on Petri dishes only after rounds of cultivation in situ (Bollmann et al., in preparation). In situ incubation seems to facilitate the appearance of domesticated variants that have fewer growth

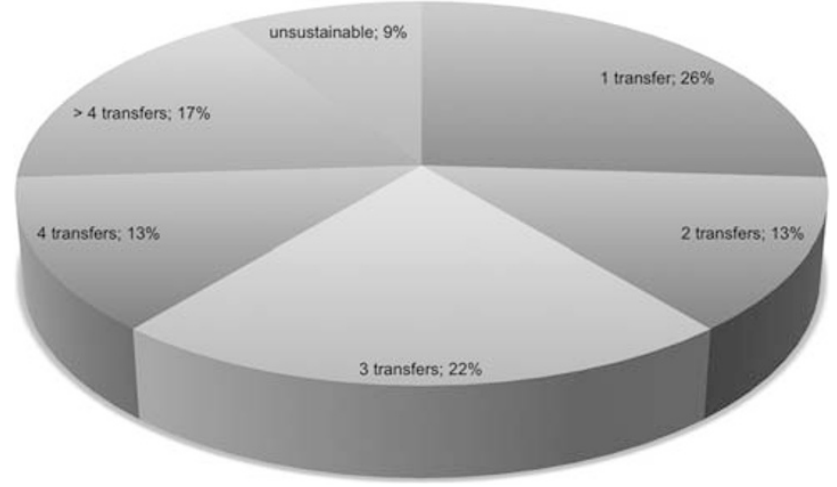

Figure 6 Percent of 'uncultivable' isolates producing domesticated variants after transferring their colonies through a series of incubations in diffusion chambers; original data from Nichols et al. ${ }^{35}$

restrictions and are capable of growing in vitro. Although the mechanism of domestication is still unknown, a substantial pool of novel microbial diversity can be accessed through this process.

\section{GROWTH FACTORS}

We noticed that some organisms forming colonies in the diffusion chamber can grow on a Petri dish, but only in the presence of other species from the same environment ${ }^{35,37}$ and suggested that uncultured bacteria only commit to division in a familiar environment, which they recognize by the presence of growth factors released by their neighbors. Interspecies symbiosis based on nutrient exchange (syntrophy) is well known in the bacterial world. ${ }^{43}$ Bacteria are also known to communicate using an interspecies quorum sensing factor AI-2 that induces synthesis of proteins that are useful for a community rather than a single cell, such as toxins or polymer hydrolases. ${ }^{44}$ Uncultured bacteria, however, do not grow on rich synthetic media, and AI-2 has not been found to function as a growth-promoting factor, raising questions about the nature of unknown growth-promoting factors in microbial communities. Co-culture provides a convenient bioassay for isolating the growth factors. For example, the described above peptide growth inducer, LQPEV, of Psychrobacter sp. MSC33, was discovered from observations of growth induction in co-culture experiments. ${ }^{37}$ Its optimal activity was observed at a concentration of $3.5 \mathrm{~nm}$, suggesting its role as a signal as opposed to a nutrient.

To assess the commonality of the growth dependence of uncultured organisms on neighboring species and pick good models for study, we chose an environment where bacteria live in a tightly packed community. ${ }^{45}$ This is a biofilm that envelopes sand particles of a tidal ocean beach (Figures $7 \mathrm{a}$ and $\mathrm{b}$ ). After disrupting the biofilm, cells were 

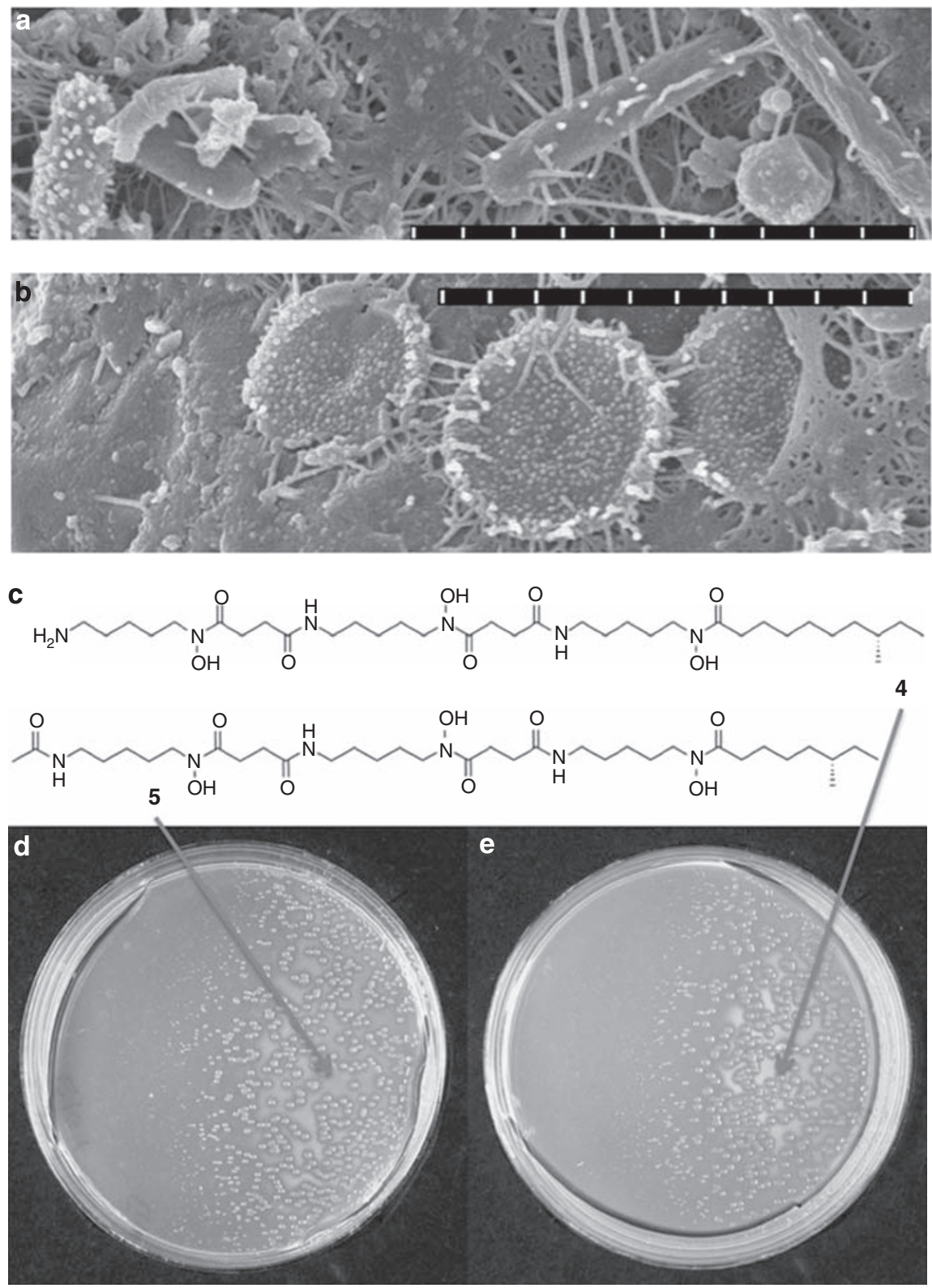

Figure 7 Uncultured bacteria growing in a sand biofilm are induced by siderophores from neighboring organisms within the biofilm. Cells of various morphology can be seen growing on the surface of a grain of sand collected from intertidal sediment (a; scale bar $=3 \mu \mathrm{m}$ ). Cells of an unidentified microbe within the biofilm with an unusual 'flat' shape (b; scale bar $=3 \mu \mathrm{m})$. Novel siderophores were purified from a model helper M. Iuteus KLE1011 that induces the growth of model unculturable M. polysiphoniae KLE1104. M. Iuteus KLE1011 Sid4 and Sid5 are shown (c) that induce growth of KLE1104 (d, e).

plated on nutrient medium containing sterile seawater (R2Asea). After 5 days of incubation at $30^{\circ} \mathrm{C}$, colonies of varied morphology appeared on the plates. There were disproportionately more colonies appearing on densely inoculated plates compared with more dilute plates. This indicated that some of the cells that grew on the densely seeded plates were receiving growth factors from neighboring colonies. To test the possible growth dependence of microorganisms on neighboring species, we picked the pairs of colonies growing within a $2 \mathrm{~cm}$ distance of each other from high-density plates (50-200 colonies per plate) and restreaked in proximity to each other. Potential uncultured isolates were identified by their diminishing growth with increasing distance from the cultivable 'helper' strain on the cross-streak plates. Colonies of the culturable organism Micrococcus luteus KLE1011 (a marine sand sediment isolate $99.5 \%$ identical to M. luteus DSM $200030^{\mathrm{T}}$ according to $16 \mathrm{~S}$ rRNA gene sequence) grew larger as their distance from other colonies increased. This pattern of growth is typical for cultivable bacteria, with the size of colonies increasing in a less crowded, nutrient-rich environment. In contrast, colonies of the uncultured isolate Maribacter polysiphoniae KLE1104 (99.9\% similar to M. polysiphoniae LMG $23671^{\mathrm{T}}$ ) became barely visible as the distance from the cultivable helper organism increased. Approximately 100 randomly picked pairs of colonies were restreaked from the high-density plates, and $10 \%$ of these pairs showed this pattern of growth induction on cross-streaked plates.

To isolate growth factors, we tested the spent medium from the helper M. luteus KLE1011 and found it to induce growth of the uncultured M. polysiphoniae KLE1104. An assay-guided fractionation led to isolation and structure determination of five siderophores and 
each of them was able to induce growth of M. polysiphoniae KLE1104 (Figures $7 \mathrm{~d}$ and e). This showed that siderophores represent the growth factors responsible for the helping activity. The siderophores consisted of a central core with alternating $N$-hydroxycadaverine and succinic acid units and were of the desferrioxamine class. ${ }^{46}$ The metabolites differed by terminal acyl attachments that drastically affect the central core's aqueous solubility. Three of the siderophores (3-5) terminate with branched chain fatty acids whereas two (1 and 2 ) terminate with an atypical phenylacetyl moiety (Figure 7c). Acyldesferrioxamines $\mathbf{1}$ and $\mathbf{3}$ were also observed, beginning with a primary amine acetyl-cap ( 2 and $\mathbf{5}$ ).

As siderophores provide bacteria with a soluble source of iron, we investigated whether drastically increased quantities of soluble elemental iron could similarly allow growth of the uncultured isolates. Iron completely oxidizes to the insoluble $\mathrm{Fe}(\mathrm{III})$ state at the $\mathrm{pH}$ and oxygen levels of both seawater (the intertidal surface sand is continually mixed by wave action) and the laboratory media; however, by adding soluble $\mathrm{FeSO}_{4}$ after the media are autoclaved, the level of bioavailable $\mathrm{Fe}(\mathrm{II})$ is temporarily increased. Incubation of $M$. polysiphoniae KLE1104 over a range of $\mathrm{Fe}(\mathrm{II})$ concentrations showed that $\sim 40 \mu \mathrm{M} \mathrm{FeSO}$ was necessary for optimal growth. Because this exceeds the reported picomolar levels of iron in the surface ocean ${ }^{47}$ by some six orders of magnitude, it is unlikely that uncomplexed iron is available to these organisms in the environment.

Given that siderophores from both $E$. coli and a neighboring species (M. luteus KLE1011) were able to induce growth of uncultured M. polysiphoniae KLE1104, it was important to determine the extent of siderophore promiscuity in growth promotion. Therefore, a screen was conducted to isolate uncultured bacteria induced by M. luteus KLE1011 and test their siderophore preferences. Environmental samples from Canoe Beach were spread on solid medium and then a culture of M. luteus KLE1011 was spotted on the plate as a potential helper. After incubation, colonies were picked and cross-streaked against $M$. luteus KLE1011. Plates were observed for a growthdependent pattern and candidate uncultured organisms were verified by seeding a plate spotted with the helper. A total of 185 colonies were screened of which 46 (25\%) were helped by M. luteus KLE1011; the rest were independently fully culturable. Twenty of the M. luteus KLE1011-dependent isolates showed weak growth when plated alone, whereas the remaining 26 completely dependent isolates ( $14 \%$ of 185 screened) had no growth in the absence of helper. Six isolates that showed strong dependence on M. luteus KLE1011 and were not capable of producing colonies on their own were chosen for further study. On the basis of 16S rRNA gene sequence comparisons, we found that the six uncultured isolates were $96.5-99.3 \%$ identical to cultivable relatives, which are typical marine bacteria.

To further examine siderophore specificity in growth induction, we tested a panel of 20 commercially available siderophores from both bacterial and fungal sources, desferrivulnibactin purified from Vibrio vulnificus and the five siderophores from M. luteus KLE1011 for their ability to induce growth of the six uncultured organisms identified in the screen. The model uncultured M. polysiphoniae KLE1104 and one other strain (KLE1009) isolated from initial cross-streaks with environmental helpers were also tested with the siderophore panel. The siderophore panel consisted of linear and cyclic trihydroxamates, dihydroxamates and carboxylic acid-type siderophores, providing a range of structural types that target different siderophore receptors. Each of the eight isolates was induced by a particular set of siderophores. KLE1009 showed wide siderophore promiscuity and was induced by all 26 siderophores tested, whereas other strains, such as KLE1123, showed greater selectivity and only grew in the presence of seven of the siderophores. Acyl-desferrioxamine 1 identified from the model helper M. luteus KLE1011 was capable of inducing growth of all eight isolates tested, whereas the variable side chains in acyl-desferrioxamines 2-5 caused a decrease in the spectrum of growth induction. A broad range of growth induction was only observed for 2 of the 16 commercial siderophores: the linear trihydroxamates desferrioxamine B (equivalent to the hydrophilic core of the M. luteus KLE1011 siderophores) and desferricoprogen. Isolates belonging to the division Bacteroidetes were able to use a wider variety than the Proteobacteria tested. The Bacteroidetes isolates were induced by an average of 22 of the siderophores, whereas the Proteobacteria were induced by an average of 10. Taken together, these data showed a considerable variation in siderophore preferences among the uncultured isolates.

The screening for uncultured microorganisms that require M. luteus KLE1011 produced various isolates, but all of them were closely related to known cultivable species. This may have resulted either from the preponderance of common microorganisms in this environment, both cultivable and uncultured, or from the bias in the screen. Given the preference for particular siderophores, we sought to obtain uncultured isolates by an unbiased approach. For this purpose unphysiologically high concentration of soluble $\mathrm{Fe}(\mathrm{II})$ was used as a surrogate for siderophores in an attempt to cultivate distant uncultured microorganisms and circumvent the natural systems that would likely require new siderophore classes.

A sample from marine sediment was cultured on a medium with a high concentration of Fe(II), and then screened for isolates that would only grow in the presence of $\mathrm{Fe}(\mathrm{II})$. Of 121 isolates, 31 showed strict dependence on $\mathrm{Fe}(\mathrm{II})$. 16S rRNA genes were sequenced for 15 of these, identifying 13 separate isolates and 2 potential duplicates. The 13 separate isolates varied in their sequence identity to typed species between 91.7 and $99.8 \%$, and the large proportion of strains isolated only once suggests that this environment has a considerable diversity of uncultured organisms. Importantly, 3 organisms in this small set of 15 were highly different from any typed strains by $16 \mathrm{~S}$ rRNA gene sequence. One is a Verrucomicrobia sp. (KLE1210), a member of a phylum that is widespread in nature but is known mainly from uncultured isolates based on direct determination of 16S rRNA gene sequences from environmental DNA. Verrucomicrobia bacterium KLE1210 is only $91.7 \%$ identical to the closest cultivable species, Rubritalea spongiae YM21-132 ${ }^{\mathrm{T}}{ }^{48,49}$ A sample from the same environment was screened for cultivable organisms able to support growth of Verrucomicrobia sp. KLE1210, as the model helper M. luteus KLE1011 was incapable of inducing growth. Intertidal sediment was added to the surface of 10 plates seeded with Verrucomicrobia sp. KLE1210 and observed for growth of bacteria from the sediment surrounded by growth of KLE1210. A candidate helper was identified, isolated and verified to induce growth of KLE1210. This led to the identification of an isolate with $97.1 \% 16 \mathrm{~S}$ sequence identity to Lacinutrix mariniflava AKS432 ${ }^{\mathrm{T}}$ (KLE1211), which is capable of inducing growth of KLE1210. Assay-guided fractionation and MS analysis of spent supernatant resulted in the detection of the siderophore growth induction factor. Although this siderophore induces growth, none of the 26 tested siderophores from the panel induced growth, revealing the high siderophore selectivity of KLE1210.

The second novel isolate, KLE1250, belongs to a recently described alpha-proteobacterial order 'Parvularculales's ${ }^{0}$ Parvularculaceae KLE1250 shows $93.7 \%$ identity by $16 \mathrm{~S}$ rRNA gene sequence to Parvularcula bermudensis KCTC $1208^{\mathrm{T}}$, one of only two initially described isolates ${ }^{51}$ that established this putative order and the only examples reported to be cultivated. The apparent rarity of this order may be due to most species being uncultured, rather than an actual 
scarcity in the environment. Isolate KLE1250 may represent a new genus within this putative order.

The third novel isolate, KLE1212, is a member of the gammaproteobacteria and shows only $90.4 \% 16$ S identity to the nearest typed strain, Kangiella aquimarina SW-154T. The isolate was cultured by plating cells resuspended from a single sand grain on media supplemented with $\mathrm{Fe}$ (II) and screening for species strongly dependent on high concentrations of $\mathrm{Fe}(\mathrm{II})$. Cultivable isolates from the biofilm of the same grain of sand were screened for their ability to induce growth of KLE1212, resulting in the identification of a helper (KLE1213) with 99.7\% 16 S identity to $V$. tasmaniensis LMG 21574T. Interestingly, none of the characterized isolates from $\mathrm{Fe}(\mathrm{II})$ plates could be induced to grow by the model helper M. luteus KLE1011, indicating both altered specificity in the growth dependence, and high diversity of uncultured species.

This study identified the first class of growth factors for uncultured bacteria and suggests that additional ones will come from analyzing organisms growing in co-culture. Perhaps the most puzzling aspect of the 'uncultured' phenomenon is the fact that many microorganisms closely related to known and well-studied cultivable species are only known from their 16S rRNA gene sequence signatures obtained directly from environmental samples. Indeed, why would bacteria closely related to organisms that thrive on a large variety of nutrients in vitro fail to grow similarly well? Our study results imply that microorganisms belonging to unrelated groups examined in this study have lost the ability to produce siderophores autonomously or fail to upregulate production when plated on synthetic media. It is difficult to unequivocally establish whether a gene was lost or was never present in the genome, however the fact that siderophores are present in all known groups of bacteria strongly suggests that siderophore dependence is a result of a secondary loss of function. This finding is surprising and highly counterintuitive from what we know about siderophore production by cultivable microorganisms. Many species can acquire siderophores they do not produce, a phenomenon known as 'siderophore piracy. ${ }^{52,53}$ For example, mycobactin and carboxymycobactin produced by Mycobacterium species induce the FemIRA siderophore transport system in P. aeruginosa. ${ }^{54}$ It appears that it is a common phenomenon for bacterial genomes to contain more siderophore uptake genes than synthesis genes. For example, genome and biochemical analyses on Pseudomonas fluorescens SBW25 showed the presence of 24 putative siderophore receptors, allowing the cell to acquire a range of heterologous pyoverdine siderophores in addition to its own. ${ }^{55}$ Siderophore synthesis is typically upregulated when available iron is low, ${ }^{56}$ but uncultured bacteria do not follow this strategy. The cost of maintaining siderophore biosynthetic genes is probably very low, on the other hand, the cost of losing the ability to produce siderophores appears imposing-inability to grow in any environment that lacks suitable siderophores. Owing to the widespread nature of siderophore synthesis in aerobes ${ }^{57}$ and the considerable level of horizontal transfer of these systems, ${ }^{58}$ it is unlikely that there is an aerobic lineage that has never possessed the ability to make them, suggesting a functional loss in uncultured organisms. A possible advantage to not being able to autonomously produce siderophores may be the security of growing in an environment populated by favorable neighbors. The specificity of siderophore uptake will then determine the range of environments suitable for growth. Growing populations are vulnerable to noxious conditions, whereas nongrowing stationary and dormant persister cells are very resilient, ${ }^{59}$ which may provide a distinct advantage for cells that only grow in familiar environments. The siderophore-dependent strains cultured by this method belong to a diverse set of bacterial groups, but further work needs to be carried out exploring the extent of siderophore depen-
Table 1 Isolates only distantly related to known relatives cultured by NovoBiotic

\begin{tabular}{lll}
\hline Isolate & Percent identity & Genus (highest match) \\
\hline L1884 & 89.3 & Steroidobacter \\
L1729 & 91.0 & lamia \\
L1287 & 91.1 & Micromonospora \\
L1491 & 91.6 & Acinetobacter \\
K0866 & 92.9 & Micromonospora \\
L1583 & 93.3 & Patulibacter \\
R1200 & 93.7 & Prosthecomicrobium \\
B2337 & 94.0 & Amycolatopsis \\
G0505 & 94.1 & Mucilaginibacter \\
K1641 & 94.5 & Actinomadura \\
P2749 & 94.6 & Paenibacillus \\
X0951 & 94.7 & Nocardioides \\
R0161 & 94.8 & Kineococcus \\
P1252 & 94.8 & Rhodococcus \\
R1227 & 94.9 & Agromyces \\
K1598 & 95.0 & Nocardia \\
G0540 & 95.0 & Methylobacillus \\
\hline & & \\
\hline
\end{tabular}

dence among members of the actinomycetes, which are well known to be the major producers of important secondary metabolites. While focusing on the culture of novel species of actinomycetes is more likely to result in the discovery of secondary metabolites, it remains to be seen whether additional rare bacterial divisions, dependent on growth factors, will be uncovered which also produce valuable metabolites.

Interestingly, this growth-dependent strategy appears very similar to the germination of spores. Recently, it was reported that germination of $B$. subtilis spores is strongly induced by muropeptides, breakdown products of peptidoglycan derived from other bacteria. ${ }^{60}$ Apparently, the presence of nutrients is not enough, and there must be other factors indicating the presence of an environment favorable for growth.

\section{PROSPECTS FOR ANTIMICROBIAL DISCOVERY}

In situ cultivation methods and the identification of growth factors for uncultured bacteria hold a distinct promise to access a previously hidden diversity of organisms for the discovery of secondary metabolites, especially the badly needed antibiotics. The diffusion chamber technology is being used by NovoBiotic (Cambridge, MA, USA), a biotech company, to grow uncultured actinomycetes as a source of new antimicrobials. Using primarily soil cultivation, it is found that the method has yielded a rich variety of organisms, including actinomycetes only distantly related to cultivable species (Table 1). Chemical novelty usually follows from biological novelty, and the promise of the uncultured organisms in antibiotic discovery should be realized within the near future. What will be of particular interest is to see whether the probability of finding novel chemistry is sufficiently greater as compared with cultivable organisms obtained from the same environment.

1 Winterberg, H. Zur Methodik der Bakterienzahlung. Zeitschr. f. Hyg. 29, 75-93 (1898). 2 Amann, J. Die direkte Zählung der Wasserbakterien mittels des Ultramikroskops. Centralbl. f. Bakteriol. 29, 381-384 (1911).

3 Staley, J. T. \& Konopka, A. Measurement of in situ activities of nonphotosynthetic microorganisms in aquatic and terrestrial habitats. Annu. Rev. Microbiol. 39, 321-346 (1985).

4 Butkevich, N. V. \& Butkevich, A. V. S. Multiplication of sea bacteria depending on the composition of the medium and on temperature. Microbiology (Moscow) 5, 322-343 (1936). 
5 Butkevich, V. S. Zür Methodik der bakterioloschen Meeresuntersuchungen und einige Angaben über die Verteilung der Bakterien im Wasser und in den Büden des Barents Meeres. Trans. Oceanogr. Inst. Moscow (in Russian with German summary). 2, 5-39 (1932).

6 Cholodny, N. Zur Methodik der quantitativen Erforschung des bakteriellen Planktons. Zentralbl. Bakteriol. Parasitenkd. Infektionskr. Hyg. A. 77, 179-193 (1929).

7 Jannasch, H. W. \& Jones, G. E. Bacterial populations in seawater as determined by different methods of enumeration. Limnol. Oceanogr. 4, 128-139 (1959).

8 Waksman, S. A. \& Hotchkiss, M. Viability of bacteria in sea water. J. Bacteriol. 33, 389-400 (1937).

9 ZoBell, C. E. Marine Microbiology: A Monograph on Hydrobacteriology, Chronica Botanica: Waltham, MA, USA, (1946).

10 Olsen, G. J., Lane, D. J., Giovannoni, S. J., Pace, N. R. \& Stahl, D. A. Microbial ecology and evolution: a ribosomal RNA approach. Annu. Rev. Microbiol. 40, 337-365 (1986).

11 Barns, S. M., Fundyga, R. E., Jeffries, M. W. \& Pace, N. R. Remarkable archaeal diversity detected in a Yellowstone National Park hot spring environment. Proc. Natl Acad. Sci. USA 91, 1609-1613 (1994).

12 DeLong, E. F. Archaea in coastal marine environments. Proc. Natl Acad. Sci. USA 89, 5685-5689 (1992).

13 Dojka, M. A., Harris, J. K. \& Pace, N. R. Expanding the known diversity and environmental distribution of an uncultured phylogenetic division of bacteria. Appl. Environ. Microbiol. 66, 1617-1621 (2000).

14 Fuhrman, J. A., McCallum, K. \& Davis, A. A. Novel major archaebacterial group from marine plankton. Nature 356, 148-149 (1992)

15 Giovannoni, S. J., Britschgi, T. B., Moyer, C. L. \& Field, K. G. Genetic diversity in Sargasso Sea bacterioplankton. Nature 345, 60-63 (1990).

16 Hugenholtz, P., Goebel, B. M. \& Pace, N. R. Impact of culture-independent studies on the emerging phylogenetic view of bacterial diversity. J. Bacteriol. 180, 4765-4774 (1998).

17 Liesack, W. \& Stackebrandt, E. Occurrence of novel groups of the domain Bacteria as revealed by analysis of genetic material isolated from an Australian terrestrial environment. J. Bacteriol. 174, 5072-5078 (1992).

18 Ravenschlag, K., Sahm, K., Pernthaler, J. \& Amann, R. High bacterial diversity in permanently cold marine sediments. Appl. Environ. Microbiol. 65, 3982-3989 (1999).

19 Ward, D. M., Weller, R. \& Bateson, M. M. 16S rRNA sequences reveal numerous uncultured microorganisms in a natural community. Nature 345, 63-65 (1990).

20 Allsopp, D., Colwell, R. R. \& Hawksworth, D. L. Microbial Diversity and Ecosystem Function, CAB International: Wallingford, UK, (1995).

21 Tiedje, J. M. Microbial diversity: of value to whom? ASM News 60, 524-525 (1994).

22 Staley, J. T., Bryant, M. P., Pfennig, N. \& Holt, J. G. (eds). Bergey's Manual of Systematic Bacteriology. 1st edn, Vol. 3, 2185-2190 (The Williams \& Wilkins Co., Baltimore, 1989).

23 Rappe, M. S. \& Giovannoni, S. J. The uncultured microbial majority. Annu. Rev. Microbiol. 57, 369-394 (2003).

24 Schloss, P. D. \& Handelsman, J. Status of the microbial census. Microbiol. Mol. Biol. Rev. 68, 686-691 (2004).

25 Colwell, R. R. \& Grimes, D. J. Nonculturable Microorganisms in the Environment, (ASM Press: Washington, DC, 2000).

26 Osburne, M. S., Grossman, T. H., August, P. R. \& MacNeil, I. A. Tapping into microbial diversity for natural products drug discovery. ASM News 66, 411-417 (2000).

27 Hurst, C. J. Divining the future of microbiology. ASM News 71, 262-263 (2005).

28 Young, P. Major microbial diversity initiative recommended. ASM News 63, 417-421 (1997).

29 Aoi, Y. et al. Hollow-fiber membrane chamber as a device for in situ environmental cultivation. Appl. Environ. Microbiol. 75, 3826-3833 (2009).

30 Bollmann, A., Lewis, K. \& Epstein, S. S. Incubation of environmental samples in a diffusion chamber increases the diversity of recovered isolates. Appl. Environ. Microbiol. 73, 6386-6390 (2007).

31 Bruns, A., Cypionka, H. \& Overmann, J. Cyclic AMP and acyl homoserine lactones increase the cultivation efficiency of heterotrophic bacteria from the central Baltic Sea. Appl. Environ. Microbiol. 68, 3978-3987 (2002).

32 Connon, S. A. \& Giovannoni, S. J. High-throughput methods for culturing microorganisms in very-low-nutrient media yield diverse new marine isolates. Appl. Environ. Microbiol. 68, 3878-3885 (2002).

33 Davis, K. E., Joseph, S. J. \& Janssen, P. H. Effects of growth medium, inoculum size, and incubation time on culturability and isolation of soil bacteria. Appl. Environ. Microbiol. 71, 826-834 (2005).
34 Ferrari, B. C., Binnerup, S. J. \& Gillings, M. Microcolony cultivation on a soil substrate membrane system selects for previously uncultured soil bacteria. Appl. Environ. Microbiol. 71, 8714-8720 (2005).

35 Kaeberlein, T., Lewis, K. \& Epstein, S. S. Isolating 'uncultivable' microorganisms in pure culture in a simulated natural environment. Science 296, 1127-1129 (2002).

36 Gavrish, E., Bollmann, A., Epstein, S. \& Lewis, K. A trap for in situ cultivation of filamentous actinobacteria. J. Microbiol. Methods. 72, 257-262 (2008).

37 Nichols, D. et al. Short peptide induces an 'uncultivable' microorganism to grow in vitro. Appl. Environ. Microbiol. 74, 4889-4897 (2008).

38 Rappe, M. S., Connon, S. A., Vergin, K. L. \& Giovannoni, S. J. Cultivation of the ubiquitous SAR11 marine bacterioplankton clade. Nature 418, 630-633 (2002).

39 Stevenson, B. S., Eichorst, S. A., Wertz, J. T., Schmidt, T. M. \& Breznak, J. A. New strategies for cultivation and detection of previously uncultured microbes. Appl. Environ. Microbiol. 70, 4748-4755 (2004).

40 Zengler, K. et al. Cultivating the uncultured. Proc. Natl Acad. Sci. USA 99, 1568115686 (2002)

41 Joseph, S. J., Hugenholtz, P., Sangwan, P., Osborne, C. A. \& Janssen, P. H. Laboratory cultivation of widespread and previously uncultured soil bacteria. Appl. Environ. Microbiol. 69, 7210-7215 (2003).

42 Nichols, D. et al. Use of ichip for high-throughput in situ cultivation of 'uncultivable' microbial species. Appl. Environ. Microbiol. 76, 2445-2450 (2010).

43 Mclnerney, M. J. et al. Physiology, ecology, phylogeny, and genomics of microorganisms capable of syntrophic metabolism. Ann. NY Acad. Sci. 1125, 58-72 (2008).

44 Williams, P., Winzer, K., Chan, W. C. \& Camara, M. Look who's talking: communication and quorum sensing in the bacterial world. Philos. Transact. R. Soc. London 362, 1119-1134 (2007).

45 D'Onofrio, A. et al. Siderophores from neighboring organisms promote the growth of uncultured bacteria. Chem. Biol. 17, 254-264 (2010).

46 Challis, G. L. A widely distributed bacterial pathway for siderophore biosynthesis independent of nonribosomal peptide synthetases. Chembiochem 6, 601-611 (2005).

47 Johnson, K. S., Gordon, R. M. \& Coale, K. H. What controls dissolved iron concentrations in the world ocean? Mar. Chem. 57, 137-161 (1997).

48 Wagner, M. \& Horn, M. The Planctomycetes, Verrucomicrobia, Chlamydiae and sister phyla comprise a superphylum with biotechnological and medical relevance. Curr. Opin. Biotechnol. 17, 241-249 (2006).

49 Hedlund, B. P., Gosink, J. J. \& Staley, J. T. Phylogeny of Prosthecobacter, the fusiform caulobacters: members of a recently discovered division of the bacteria. Int. J. Syst. Bacteriol. 46, 960-966 (1996).

50 Lee, K. B. et al. The hierarchical system of the 'Alphaproteobacteria': description of Hyphomonadaceae fam. nov., Xanthobacteraceae fam. nov. and Erythrobacteraceae fam. nov. Int. J. Syst. Evol. Microbiol. 55(Part 5), 1907-1919 (2005).

51 Cho, J. C. \& Giovannoni, S. J. Parvularcula bermudensis gen. nov., sp. nov., a marine bacterium that forms a deep branch in the alpha-Proteobacteria. Int. J. Syst. Evol. Microbiol. 53(Part 4), 1031-1036 (2003).

52 Luckey, M., Pollack, J. R., Wayne, R., Ames, B. N. \& Neilands, J. B. Iron uptake in Salmonella typhimurium: utilization of exogenous siderochromes as iron carriers. J. Bacteriol. 111, 731-738 (1972).

53 Schubert, S., Fischer, D. \& Heesemann, J. Ferric enterochelin transport in Yersinia enterocolitica: molecular and evolutionary aspects. J. Bacteriol. 181, 6387-6395 (1999).

54 Llamas, M. A. et al. The heterologous siderophores ferrioxamine B and ferrichrome activate signaling pathways in Pseudomonas aeruginosa. J. Bacteriol. 188, 18821891 (2006).

55 Moon, C. D. et al. Genomic, genetic and structural analysis of pyoverdine-mediated iron acquisition in the plant growth-promoting bacterium Pseudomonas fluorescens SBW25. BMC Microbiol. 8, 7 (2008).

56 Winkelmann, G. Microbial siderophore-mediated transport. Biochem. Soc. Transact. 30, 691-696 (2002).

$57 \mathrm{Kraemer}, \mathrm{S}$. Iron oxide dissolution and solubility in the presence of siderophores. Aquat. Sci. 66, 3-18 (2004).

58 Challis, G. L. \& Hopwood, D. A. Synergy and contingency as driving forces for the evolution of multiple secondary metabolite production by Streptomyces species. Proc. Natl Acad. Sci. USA 100 (Suppl 2), 14555-14561 (2003).

59 Lewis, K. Persister cells, dormancy and infectious disease. Nat. Rev. Microbiol. 5, 48-56 (2007).

60 Shah, I. M., Laaberki, M.- H., Popham, D. L. \& Dworkin, J. A eukaryotic-like Ser/Thr kinase signals bacteria to exit dormancy in response to peptidoglycan fragments. Cell 135, 486-496 (2008). 